\title{
EVALUATION OF CLINICAL PATHWAYS OF AN EMERGENCY HOSPITAL IN CASE OF AN ACUTE MASS TRAUMA ACCIDENT
}

\author{
By \\ Farrag S.I.* and Kamel E.A.** \\ * Dept. of Biomedical Engineering - Faculty of Engineering - Modern University \\ ** Occupational and Environmental Medicine - Dept. of Community Medicine and \\ Public Health - Faculty of Medicine - Mansoura University
}

\begin{abstract}
:
The ability to adapt and utilize emergency facilities is a critical element in responding to surges resulting from man-made and natural events resulting in mass casualties. Emergency services must be adequately prepared to effectively absorb a sudden increase in patients and to allow the medical staff to operate effectively

Objectives : To qualitatively describe patient, hospital care, and clinical pathway characteristics that may be associated with pathway effectiveness in an emergency department .

Methods :The current study is retrospective, cross-sectional and descriptive study the flow of clinical pathways in an emergency hospital is described in case of an acute mass trauma accident involving victims. Drawbacks and pathway laggings and redundancies were presented and discussed. A list of characteristics that might impact clinical pathway effectiveness was developed .A hypothesis-driven qualitative analysis was used to describe key characteristics that might differentiate effective from ineffective critical pathways.

Results: The care pathway adopted during the discrete event under study was perceived to be redundant by the management and by the working staff. Laggings of sequential steps and interventions were inevitable. The patient total times in the department was affected and had effects on medical services performance .
\end{abstract}


Conclusions: Recommendations for improving the effectiveness of the emergency services of the hospital under study were given. The adoption of clinical pathways may be a very promising method to improve the emergency department service at a hospital and so to react to the challenges of massive surges in patient care. Principles of care maps when integrated into the early stage of planning and design of a building and a service are expected to facilitate modifications to be implemented at modest capital cost increases for the benefit of both high-risk patients and over-stressed Health Care Workers.

Keywords: Clinical pathways , mass trauma, emergency, control of injuries

\section{Introduction}

Clinical pathways, referred to as care pathways, critical pathways, integrated care pathways or care maps are methods for the patient-care management of a welldefined group of patients during a welldefined period of time ${ }^{[4]}$. The ultimate goal is to preserve quality of care, reduce risks, increase patient and staff satisfaction and increase the efficiency in the use of resources taking into account costeffectiveness of care.

Care pathways may be represented by a flow chart format of the decisions to be made and the care to be provided for a given patient or patient group in a given condition in a step-wise sequence. They are structured, multidisciplinary plans of care designed to support the implementation of clinical guidelines and protocols. Meanwhile, clinical pathways differ from practice guidelines and protocols in the fact that they are intended to be utilized by a multidisciplinary team and have a focus on the quality and co-ordination of care ${ }^{[2]}$.

One important discrete event for a clinical pathway to prove effective would be in case of an emergency situation which results in mass casualties and a surge in patient care. This situation would be confronted in case of natural or man-made disasters, epidemics or serial highway traffic accidents, for example. In these situations, medical services are expected to be offered efficiently and effectively to a significant influx of high-risk patients ${ }^{[10]}$.

It is perceived as extremely stressful for medical facilities annexed to occupational health departments of major industries as chemical and military industries to face an acute mass trauma accident resulting in major injuries and mass casualties ${ }^{[5]}$. Such situations are rare to occur but constitute an immense challenge for medical facilities 
to confront; prior risk assessments and contingency plans must be prepared. The medical facilities should be capable to extend their services in such situations in ultra short time. In this instance, the optimum clinical pathway would offer much help to victims of the accidents and would assist healthcare workers in offering emergency services. The ultimate outcome would be better mortality and morbidity statistics among workers population involved in the accident. Such major accident could be confronted in a multitude of other situations involving other groups of victims as in case of injuries resulting from road traffic accidents.

The clinical pathway characteristics associated with pathway effectiveness in an emergency department or an emergency hospital in case of surges following acute mass trauma incidents would naturally include :- the patients' flows to- and fromthe reception area, the patients' flows through the rest of the medical facility, the staffing, the resources usage, the timing or sequencing of interventions, etc ${ }^{[3]}$. The architectural design of the medical facility is a challenge that should balance the possible risks of major events and the medical equipment design, location, mobility and access and would constitute an inseparable part of an effective clinical pathway as well. All of the just-mentioned characteristics constitute vital aspects of the multifaceted nature of an integrated care pathway. All should prove effectiveness and efficiency with outcome indicators including patient survival rates, mean time of post-interference stay in hospital, and good prognostic medical condition of treated cases, besides others. ${ }^{[8]}$

Integrated care pathways would naturally function best if the built environment of the medical facility and the layout and location of medical equipment is backing the flowchart of medical services provided. It is of utmost importance to consider the rapidity with which access is made to needed medical equipment for performing the necessary investigations for critical patients in mass trauma accidents. The medical equipment would be of no use if it does not fulfill the objective of its purchase specifically in emergency departments or medical facilities. It does not matter how new and recent the medical equipment would be if access to its use is not rapid enough to rescue lives of patients in case of emergencies. The same applies to hospital architectural design as well. It is no use to test for the clinical pathways if the venue in which they are applied and adopted is not fulfilling its proposed role. 
The fact that the adoption of efficient clinical pathways would improve the clinical care in case of emergency situations is discussed in the present research. The aim would be to analyze and criticize the actual pathway followed during a life-saving emergency situation resulting in an acute surge of critical highrisk patients with an ultimate goal to give recommendations for modifications of the clinical pathway amenable for use in future similar situations in the medical facility selected as a case-study. The adoption of these recommendations could be tested prospectively in similar situations and could be implemented during the construction and functioning of new emergency medical facilities.

It is well-known that Egypt suffers from a sustained rise in acute traumatic injuries and fatalities resulting from mass road traffic accidents specifically on highways and ring roads. It is an accepted medical fact as well that the rapidity with which medical care is offered to victims is crucial in improving survival and in improving clinical prognosis of victims. For these reasons, it was appealing to study the concept of care pathways and their potential effectiveness in a real life accident involving mass trauma and casualties on the ring road of Greater Cairo.

\section{Materials and Methods}

The following study is retrospective, cross-sectional and descriptive. The medical facility which was selected as a case-study was the National Bank of Egypt Hospital on the Ring Road near New Cairo with in-patients capacity of 84 beds. The main goal for its construction was to deal with emergency situations involving casualties in case of road traffic accidents occurring in its catchment area. This hospital is the only one of its prototype present in a circle of 10 kilometers. It will serve new communities such as ElMearag City, Zahraa El-Maadi residential area, Ebad El-Rahman City, industrial area, Katamia and Suez-katamia, highway.

The hospital plan was designed for 84 bedrooms, ground, first and second floors.

The ground floor includes Emergency department, outpatient department and service department .

The first floor includes bedrooms.

The second floor includes 3 operation theatres with their services, ICU and bedrooms .

The discrete event selected to prove the effectiveness of the clinical pathways adopted in the hospital in response to the surge was a serial accident on the ring road 
resulting in mass casualties mounting up to 66 subjects. Of them, 14 passed away on the scene of accident and 52 were transferred to the Emergency Hospital of the national Bank of Egypt. The transferred victims suffered mostly from acute traumatic injuries of various severity and from other acute potentially-fatal conditions as internal bleeding. Of them, 50 subjects survived and 2 subjects passed away.

The main method adopted was tracking the hospital's information system for detailed information on sequence of events and timing of interventions adopted during the accident. Where recorded information was lacking, tracking was done by personal communication with the management team and the staff-physicians. An approximate scenario of what actually happened was rebuilt. The comments of management and staff were recorded. The clinical pathway was reconstructed, then qualitatively analyzed and criticized. Comparisons with a standard simulation clinical pathway were done.

The second method was a detailed architectural study of the layout of departments and wards including the reception area and the ambulance facilities. Modifications in architectural design and medical equipment layout were proposed for utmost functioning of the simulation clinical pathway which if they have been adopted, the timing or sequencing of interventions would have improved and consequently the survival rates of victims of the accident would have been better.

\section{Results and Discussion}

\section{I- The Event description :- Two} major road traffic accidents occurred in the neighborhood of the hospital on the ring road simultaneously. All the victims whether injured or dead were transferred to the case-study hospital. About $30 \%$ of the injured cases had head injuries and compound bone fractures which could be classified as major injuries, around $45 \%$ of the injured cases had compound and simple bone fractures which could be classified as moderate-severity injuries and $25 \%$ of the injured cases had superficial wounds and musculoskeletal injuries which could be classified as minor injuries. The emergency reception ward of the case-study hospital had only six beds, with extra three beds at the emergency intensive care unit (ICU). Most of the patients had to undergo computed tomography (CT) to diagnose internal hemorrhages. The CT procedure took at least 30 minutes for each patient including patient transfer to the $\mathrm{CT}$ room and performing the CT scan. It was an unfortunate event where 52 patients were transferred to a hospital with an emergency 
reception capacity of 9 beds at most after using the ICU emergency beds. The rest of the transferred patients waited on the ambulatory stretcher in the reception area until the doctors finished the emergency care, then the admission or discharge of the first treated 9 cases. The rest of patients were offered treatment successively in 9-patients' batches. The total time for offering emergency care, then admission, transfer or discharge of the 52 patients took about 6 hours.

\section{II- Description of the clinical / care} pathway followed post-event :- As described, there was a surge of 52 patients arriving at the emergency department of the case-study hospital which clearly imposed a high stress load on patients and on the medical staff. The actual patient flow scenario was as follows:- patients arrived at the emergency reception by the ambulance, underwent a preliminary examination and sorting. Emergency clinical care was offered, then for the major injuries and some of the moderate-severity injuries, doctors requested $\mathrm{CT}$ examinations while for others, plain X-ray was requested, which took a lot of time taking into considerations the diversity of the types of injuries. Patients who had to undergo surgical operations or be transferred to the ICU have been transferred in 10 minutes to the second floor at the operation theater suite which had three operation theaters or to the ICU.

The sequencing of interventions constituting the clinical pathway adopted during the mass trauma accident causing a surge of high-risk patients included the following steps :-

- Notification of the ambulance Dept of the hospital.

- Arrival of victims into the emergency reception ward.

- Preliminary examination and triage of cases according to type of apparent injuries.

- First aid treatment provision.

Detailed examination by specialty physicians on call.

- Referral of cases for CT and transfer toand from- reception ward.

- Referral of other cases for plain X-ray and transfer to- and from-reception ward.

- Transfer of patients to operation theaters where emergency operations were done.

- Transfer of patients to the surgery suite in-patients ward.

- Transfer of patients to orthopedic suite in-patients ward. 
- Discharge of patients from the emergency ward designated to be out-patients and treated as day-cases.

- Communication of staff and co-ordination of care althrough the previous steps.

- Dynamics of patient flows and resource usage althrough the previous steps.

- Risk assessment, management and communication to staff.

- Transfer of passed-away patients to the morgue.

III- Qualitative analysis of the clinical / care pathway followed postevent :-

Tracking the steps of the clinical pathway, it becomes evident that biomedical equipment design, location and ease of access would constitute a fundamental aspect of an effective functional care pathway. Architectural design and human resources availability constitute another vital and crucial facet of the clinical pathway as well. Time and timing of interventions are critical in case of emergency medical care in acute mass trauma victims. It is calculated in seconds and minutes rather than in hours.

The care pathway adopted during the discrete event under study was perceived to be redundant by the management and by the working staff. Laggings of sequential steps and interventions were inevitable. Marked flow jams in patients' transfer were observed. Both working medical and paramedical personnel and the management staff felt that time would have been more efficiently employed if certain modifications would have been applied. The outcome would have been observed on the prognosis of patients after the event as percentage of victims who survived, length of time of stay in the hospital, percentage of temporary disability and percentage of permanent disability post-event.

The pitfalls in the emergency response of the case-study hospital and in the medical care offered to the patients could be attributed to some of the following causes :-

- The Emergency Surgery Room at the ground floor was not routinely used at all during any surgical interventions at the hospital whether for sporadic cases or in case of surge of high-risk patients on exceptional days because the doctors did not like to use it. This was attributed to the poor layout of this surgery room as it did not have a recovery room annexed to it and had poor equipment design as well. Consequently, all emergency patients needing surgical intervention had to be transferred to the operation theaters at the second floor. The net result was that the hospital had 
practically-speaking lost a valuable space of the emergency department that was occupied by the emergency surgery room. Design and ergonomics were both defective.

- The case-study hospital that was originally-designed containing 84 beds, did not include a central sterilization unit and its morgue could not accommodate more than 3 bodies without refrigeration (please note that there was 16 dead bodies).

- It is a must that any emergency department should include an oxygen-line, suction devices, medical air. The case-study hospital did not have a central gas network and the emergency department was not supplied by an oxygen-line which is an important factor of the resuscitation equipment in case of surge of high-risk patients.

- It was noticed that there was a special defibrillator room around 20 meters from the emergency department whereas the emergency department should include within its premises at least one defibrillator, several ECG machines, Monitors with Non invasive blood pressure (NIBP) and $\mathrm{SpO} 2$. and a Ventilator. Therapeutic gases should be internally piped to all patient care areas,

- The concept of clinical pathways was lacking, which if applied correctly and evaluated routinely would certainly improve the flow of patients specifically in case of surge of high-risk patients in emergency situations.

- Training of emergency staff on simulation scenarios of emergency events was lacking . A hospital Incident Command System should be developed as well as an Emergency Preparedness program.

\section{Recommendations}

\section{I- Requirements and guidelines for} planning an emergency department :-

The emergency department should be planned by zones, and should follow the functional relationships summarized by the following diagram ${ }^{[1],[6-7],[9]:-}$

\section{EMERGENCY DEPARTMENT}

\begin{tabular}{|l|l|l|}
\hline Direct Access * & Ready Access ** & Access*** \\
\hline & Coronary Care Unit & Inpatient wards \\
& Intensive Care Unit & Pharmacy \\
Medical Imaging & Operating Theatre & Mortuary \\
& Pathology/Blood Bank \\
& Medical Records & \\
\hline
\end{tabular}


1- The first Zone* : Direct access, that means immediate access . i.e. Medical imaging should constitute an inseparable part of the emergency department.

2- The second zone** : Ready access, means rapid and easy access, i.e. ICU should be located directly above the emergency department with a special lift from the emergency department to the ICU.

3- The third zone*** : Access, means the ability to reach the zone and use the medical services with no time pressure.

\section{Medical Imaging :}

Immediate access to CT scanning, and plain X-ray modalities will enhance the emergency department's effectiveness. A system of electronic display of imaging is desirable.

\section{Intensive Care Unit and Coronary Care} Unit :

Rapid access is highly desirable to minimize transfer time of critically ill patients. It should be located in the first floor vertically over the emergency department.

\section{Operating Theater :}

Rapid access is highly desirable in certain surgical emergencies.

\section{Laboratory :}

Rapid access is highly desirable to minimize time to laboratory investigations.

\section{Design considerations of the emergency}

\section{department :}

It should allow rapid access to every space with a minimum of cross traffic. There must be close proximity between the Resuscitation / Acute Treatment areas for non-ambulant patients and other treatment areas for ambulant patients, as staff may require relocation at times of high workload.

\section{Site Selection :}

Decisions regarding site location have a major influence on the eventual cost and operational efficiency of the department and should be made in consultation with emergency department staff. The site of the emergency department should, as much as possible, maximize the choices of layout. In particular, sites of access points must be carefully considered.

\section{Description of ideal patient flow :}

\section{A- Triage}

Patients may present self-referred or via ambulance. All patients should be triaged through a single point. The aim of triage is to "sort" patients in order to provide 
optimum care consistent with their medical need and to ensure the efficient utilization of the available resources. After triage, patient details are recorded by the clerical staff and a medical record is either initiated or a previous medical record retrieved.

\section{B- Treatment}

Patients may be directed to:
a. The Resuscitation area
b. The Acute Treatment area
c. The Consultation area
d. Medical Imaging
e. Waiting area

In cases a. - c., consultation/ examination/investigation/treatment will be performed either in sequence or concurrently, depending on the severity of the patient's condition. Support services and, in certain cases, specialized areas, eg. plaster room, may be utilized. After treatment, patients are either admitted, transferred or discharged.

\section{C- Resuscitation Room}

This room is used for the resuscitation and treatment of critically ill or injured patients. It has the following requirements:

The resuscitation area should be easily accessible from the ambulance entrance and separate from patient circulation areas and must be easily accessible from the staff station in the Acute Treatment/ Observation area. The Resuscitation area should have a full range of physiological monitoring surgical light, defibrillator , and resuscitation equipment such as Infusion pumps, Blood warmer, Portable ventilator, and Resuscitation trolley with X-Ray capacity, CPAP/BiPAP Machine.

\section{D- Plaster Room}

The Plaster room allows for the application of Plaster of Paris and for the closed reduction under sedative, or anaesthesia, of displaced fractures or dislocations. It must be at least $16 \mathrm{~m} 2$ in size, excluding crutch or splint storage areas. Provision for physiological monitoring during procedures will be necessary.

\section{II- Modifications proposed to the case- study hospital :}

For the ground floor :- Emergency care should follow this circulation :First the entrance of the patient from the ambulance to the emergency boxes, where the patient will be observed and then will be transferred easily to the hospital departments of orthopedics , surgery , ...etc. The emergency operation theatre should include its own recovery room which should be 
connected to the main operation theater in horizontal direction in order to save patient life. It is preferred to plan the location of the main operation theaters on the ground floor which will provide a smooth circulation for the emergency patients and will have easy connection with the service departments located at the basement as well.

For the emergency surgery room, it should include a recovery room, it is suggested to use the room adjacent to it, which is used for observation for recovery as well.

The ICU should be located on the first floor above the emergency department with special lift for immediate access.

Training of medical and paramedical staff using simulation models for clinical pathways would be of help in future surge events. Proposed future scenarios of probable events are to be built through tracking of past incidents and using wellknown Hospital Incidents Command Systems.

\section{Conclusion}

In the present research, modifications of clinical pathways in an emergency situation were qualitatively described. The three areas on which the design of emergency departments should be based are the capability, capacity and protection of health care workers in the departments. Planning for Disaster Preparedness should begin before the hospital is designed rather than modifications being recommended thereafter.

Evaluating threats that are present in the region will serve to determine the type of protection, capability and capacity needed. Another concept is to ensure that key services and additional usable space are located close enough to the emergency department to reduce the logistics effort during handling a disaster situation medically.

Careful planning can result in a greater capacity and likely better outcomes in time of disaster without imposing excessive contingency space or cost requirements. Furthermore many of the features that enable the emergency department to function in these critical situations can also enhance capabilities during routine functioning.

\section{References}

1. ACHS Accreditation Guide, Standards for Australian Health Care Facilities, (1995).

2. Blaser R, Schnabel M, Biber C, Baumlein M, Heger O and Beyer M. (2007). Improving pathway compliance and clinician performance by using information technology. Int J Med Informatics, 76:151-56.

3. Cardeon B, and Demeulemeester E (2008); Capacity of clinical pathways - a strategic multi level evaluation tool ; J.Med Syst. 32; 443-52 
4. De Bleser L, Depreitere R, et al. , (2006). Defining pathways. J N. Management, 14: 553- 63.

5. Delphine R, Marc C. et al. ; (2008) Managing an emergency department by analysing HIS medical data :a focus on elderly patient clinical pathways; Health Care Manage Sci 11:139-46.

6. Emergency Unit Design Guidelines, Health Department of Western Australia Facilities Unit, (2008).

7. Guidelines for design and construction of hospital and healthcare facilities ; The American Institute of Architects (2008).
8. Keon-Hyung L., Seung-Bum Y. et al. (2009) ; The Association between Hospital Ownership and Technical Efficiency in a Managed Care Environment; J Med Syst 33:307-15.

9. Riggs JR (1993), Emergency Department Design. American College of Emergency Physicians.

10. Zilm F, Berry R, et al.(2008). Integrating disaster preparedness and surge capacity in emergency facility planning. J Amb.Care Mana. 31; 4: 377-85. 